\title{
Bestandtheile des zu Salzungen im Herzogthume Meiningen und zu Stotternheim im Grossher- zogthume Weimar zum Salzsieden und Baden verwendeten erbohrten Soolwassers;
}

von

H. W a clien roder.

In einer früheren ausführlichen Abhandlung (S. dieses Archiv Bd. 17. 1839) über die Salzunger Soole habe ich dargethan, dass die Thüringischen Salzquellen das reinste Kochsalz liefern, welches überhaupt producirt wird. Die Soolen kommen ohne Zweifel aus gleichen Gebirgsmassen, aus dem Schlottengyps und salzhaltigen Thon der Zechsteinformation, und gehen öfters aus dem Mergel der Formation des bunten Sandsteins zu Tage aus, sind aber so geringhaltig, dass sie bis dahin durch Gradiren siedwürdig gemacht werden mussten. In neuester Zeit aber hat man auf mehreren Salinen Thüringens die Unkosten, ein Bohrloch niederzutreiben, nicht gescheuet. Die Versuche sind mit bestem Erfolge gekrönt worden zu Artern, Salzungen*)

*) Ueber den Bohrversuch der Saline zu Salzungen hat Herr Salzgraf Schüler zu Salzungen mir cinen Bericht mitgetheilt, aus welchem ich Folgendes aushebe. Der Bohrversuch währte vom August 1840 bis Januar 1842. Nach Durchbohrung von Dammerde und Kiesellagern, zusammen 12 Fuss mächtig, kam man auf bunten Sandstein, welcher sich bis zu 218 Fuss Teufe fortsetzte. Hier erschien wechsellagernd rother Schieferletten, Knollen von Anhydrit und Chlorit, 14 Fuss mächtig, sodann ein kalkhaltiger Sandmergel und blauer Thon. Bei 247 Fuss Teufe brach ein 5,3 proc. Soolwasser mit 20 Fuss Ueberdruck hervop. Nach Einsenkung von Röhren in diesen artesischen Brunnen gelangte man auf ein 56 Fuss mächtiges Lager sehr festen Stincksteins der Zechsteinformation, in welchem auch dio Quelle sich bedeutend senlite. Hierauf folgte graulicher körniger Schlottengyps mit Thon, in welcher Gebirgsmasse das Bohrlock ondet. Die zuletzt durchbohrten Mlassen bestehen in einem mit Salz durchwachsenen Thonlager und in einem unreinen, mit Thon und Fasergyps durchwachsenen Steinsalz. Die voll gesïlligte Soolc 
und Stotternheim. An andern Orten, wie zu Sulza, wird das Bohren noch fortgesetzt. Die erbohrten Soolen sind vollständig gesättigte Salzlösungen und können daher sogleich versotten werden. Die Soolen werden durch Pumpen aus der Tiefe herausgehoben.

Da die Soole von Salzungen schon vor langer Zeit zugleich als Heilquelle benutzt worden und gegenwärtig in der dort vortrefflich eingerichteten Badeanstalt von einer grossen Anzahl Badegaisten alljährlich mit bestem Erfolge benutzt wird, so erschien es der Plännerschaft und Badedirection zu Salzungen wünschenswerth, die gegenwärtig allein verwendete erbohrte Soole eben so genau chemisch kennen zu lernen, als dic fruhere schwächere Salzquelle.

Die erbohrte Soole zu Stotternheim wird ebenfalls nicht bloss zum Ausbringen eines sehr reinen Kochsalzes, sondern auch in der Nähe der Saline, namentlich in der Stadt Weimar, zuweilen zum Baden verwendet. Ich glaube daher die Resultate der chemischen Analyse dieser Soole hinzufügen zu dürfen.

Das spec. Gewicht der Salzunger Soole ist bei $+8^{\circ} \mathrm{C}$ $=1,2065$, das der Stotternheimer Soole $=1,2032$. Schon hieran erkennt man die last völlige Gleichheit beider Soolen.

1) Der trockne feste Salzrickstand, den die Soolen beim Abdampfen hinterlassen, beträgt bei der ersteren 26,87 Proc., bei der letztern 26,653 Proc. Beim Auflösen des stark getrockneten Salzrickstandes der Salzunger Soole hinterbleibt nur 0,3 6 Proc. Unlösliches zurück, bestehend aus 0,040 Proc. Kieselerde, 0,008 Proc. Kalk und 0,04 Proc. Talkerde. Das Salzgemenge aus der Stotternheimer Soole löst sich bis auf eine unbedeutende Trübung völlig klar wieder in Wasser auf.

2) Der Salzrückstand der Salzunger Soole mit wässerigem Weingeist mehrere Malc ausgezogen, die Flüssigkeit eingeengt und mit Platinchlorid versetzt, gab so viel

wird mit ciner Pumpe aus 482 Fuss licfe herausgehoben, und schlägt bei $90^{\circ}$ R. Kochsalz nieder. 
Kaliumplatinchlorid, dass 0,149 Proc. Kali oder 0,235̌6 Proc. Chlorkalium daraus berechnet werden konnten. In der Stotternheimer Soole konnte aber ein Gehalt an Kali nicht entdeckt werden.

3) Aus einer abgewogenen Menge der Soolen wurde durch oxalsaures Kali der Kalk vollständig gefällt. $\mathbf{E r}$ betrug für die Salzunger Soole $=0,434$ Proc., für die Stotternheimer $=0,0801$ Proc.

4) Aus den vom Kalk befreieten Soolen wurde durch basisches phosphorsaures Ammoniak die Talkerde quantitativ bestimmt. Der geglühete Niederschlag wurde mit 38 Proc. reiner Talkerde berechnet. In der Salzunger Soole finden sich 0,042 Proc., in der Stotternheimer 0,0459 Proc. Talkerde.

5) Die mit einigen Tropfen Salpetersäure angesäuerten Soolen gaben mit Chlorbaryum einen Niederschlag von schwefelsaurem Baryt, aus welchem für die Salzunger Soole 0,262 Proc., für die Stotternheimer aber $=0,2339$ Proc. Schwefelsäure berechnet wurden. Die Schwefelsäure wurde auf schwefelsauren Kalk und der etwa vorhandene Uchorschuss derselben auf schwefelsaures Natron, wo aber Kalk uiberschuissig war, dieser auf Chlorcalcium berechnet.

6) Einige Gramm von beiden Soolen mit der 30fachen Menge Wasser verdiunnt und mit wenig Salpetersäurc angesäuert, wurden mit hinreichendem salpetersaurem Silberoxyd versetzt. Die Niederschläge waren Chlorsilber mit einer Spur Bromsilber. In der Salzunger Soole finden sich 15,53 Proc., in der Stotternheimer $=15,67$ Proc. Chlor.

7) Die Bestimmung des Broms geschah nach der in diesem Archiv $\mathrm{Bd}$. 17. angeführten Methode; jedoch ist nur in dem Salzunger Soolwasser das Brom quantitativ bestimmt und als Bromnatrium berechnet worden, da die Stotternheimer Soole nur sehr geringe Spuren davon enthält. Das Brom beträgt in 100 Theilen der Salzunger Soole 0,0452 Theile.

8) Die Menge des Chlornatriums wurde durch Rechnung gefunden nach der genau ermittelten Menge des Chlors. Nachdem das Kali und die Talkerde auf Chloride 
270 Wackenroder, Bestandtheile von erbohrtem Soolwasser.

berechnet worden, blieben für das Salzunger Wasser 15,4147 Proc, Chlor übrig, welche 25,5454 Proc. Chlornatrium entsprechen. Im Stotternheimer Wasser sind 25,090 Proc. Chlornatrium enthalten.

Die Analyse der Salzunger Soole ist unter meiner Leitung und Mitwirkung von mehreren meiner Zuhörer mehrmals angestellt, und vornehmlich vom Hrn. Wilken aus Lübeck, Grïne aus Dresden und Ruickoldt aus Weimar mit übereinstimmendem Erfolge wiederholt worden. Bei der Analyse der Stotternheimer Soole ist aber Hr. Ruick oldt allein thätig gewesen.

Nach diesen Analysen sind enthalten in $\mathbf{1 0 0}$ Theilen der Salzunger Soole. Stotternheimer Soole.

\begin{tabular}{|c|c|}
\hline Chlornatrium........25,545t & 25,0900 \\
\hline Bromnatrium $\ldots \ldots \ldots, 0,0586$ & Spuren \\
\hline Schwefelsaures Natron . 0,1388 & $\sim$ \\
\hline Chlorkalium . . . . . . 0,2356 & - \\
\hline Chlorcalcium........ - & 0,1572 \\
\hline Chlormagnesium. . . . . 0,0047 & 0,1068 \\
\hline Schwefelsaurer Kalk ... 0,3034 & 0,4001 \\
\hline fiohlensaurer Kalk .... 0,0142 & Spuren \\
\hline Kohlensaure Talkerde... 0,0827 & - \\
\hline Kieselerde ......... 0,0400 & - \\
\hline 26,4234 & $25,7541$. \\
\hline
\end{tabular}

Die Uebereinstimmung zwischen der Summe der quantitativ bestimmten Bestandtheile und dem durch Abdampfen der Soolen direct erhaltenen Salzrickstande ist so gross, als sie überhaupt sein kann. Die Differenz ist bei der Stotternheimer Soole am grössten, und es folgt daraus, dass der Salzrückstand weniger stark erhitzt worden. Bei einer stärkeren Erhitzung würde aber der Salzrückstand auch wohl einen geringen unlöslichen Rückstand von kohlensaurer Kalk - und Talkerde hinterlassen haben, gleichwie die Salzunger Soole, deren Salzrückstand auch einige Male von reinem Wasser fast ganz klar wieder aufgenommen wurde. Dann aber würde die kleine Menge von Chlorcalcium unter den Bestandtheilen der Stotternheimer Soole vielleicht verschwinden. Kann überhaupt nicht viel Gewicht gelegt werden auf so geringe Mengen berechneter Salze 
Bauman, Kenntniss des Liebensteiner Mineralwassers. 271

in den Mineralwässern als Salzgemischen, so berechtigt doch auch nichts zu einer willkürlichen Abänderung der Ergebnisse genau durchgeführter Analysen.

\section{Beitrag zur Kenntniss des Liebensteiner Vineralwassers;}

\section{von \\ Dr. H. Baumann.}

Die Mineralquelle zu Liebenstein im Herzogthum S.- Mejningen ist öfters und zu verschiedenen 7eiten chemischen Prüfungen unterworien und bei erneuerten Analysen stets complicirter zusammengesetzt gefunden worden. Die neuesten und genauesten Analysen sind von Irn. Hofrath Dr. Wacken roder, welcher das Wasser im Jahre 4831 untersuchte und die Untersuchung im Jahre 1836 an der Quelle selbst wiederholte. Die Resultate dieser Analyse, so wie die älterer Untersuchungen von Güttling, Hoffmann und Trommsdorff findet man nebst dem Verfahren, dessen Wackenroder sich zur Ausmittelung und Abscheidung der einzelnen Bestandtheile bediente, aufgezeichnet in einer besonderen Abhandlung „chemische Analyse der Mineralquelle zu Liebenstein im Herzogthume S.-Meiningen" in dem "Neuen Jahrbnche der Chemie und Physik Bd. V. 1832. H. 9. pag. 1-47" welche auch, nebst der im Jahre 1836 ausgefuhrten Analyse in besonderem Abdruck im Buchhandel erschienen ist.

Nach der Analyse Wackenroder's enthält das Liebensteiner Mineralwasser:

im wasserleeren Zustande: in $1000 \mathrm{Th}$. in $16 \mathrm{Unzen} \mathrm{M.} \mathrm{Gew.}$

Schwefelsaures Natron ............0,18086

Kohlensaures Natron............,0,02069

Chlomatrium ..................0,16757

Chlorkalium .................,0,02136

Schwefelsaure Talkerde ...........0,04145

Chlormagnesium................0,10516

Schwefelsauren Kalk ...........,0,03182

Kohlensauren Kalk ............. 0,56700

1,3890 Gran

0,1589 "

$1,2869 \quad "$

$0,1641 \%$

0,3183 "

$0,8076 "$

0,2444,

4,3546 , 